\title{
Analysis of the spatial distribution of Aedes albopictus in an urban area of Shanghai, China
}

\author{
Yibin Zhou ${ }^{1+}$, Hongxia Liu ${ }^{1+}$, Peien Leng ${ }^{1}$, Jiang Zhu', Shenjun Yao ${ }^{2}$, Yiyi Zhu ${ }^{1 *}$ (D) and Huanyu Wu ${ }^{1 *}$
}

\begin{abstract}
Background: Aedes albopictus is a vector of major arboviral diseases and a primary pest in tropical and temperate regions of China. In most cities of China, the current monitoring system for the spread of Ae. albopictus is based on the subdistrict scale and does not consider spatial distribution for analysis of species density. Thus, the system is not sufficiently accurate for epidemic investigations, especially in large cities.

Methods: This study used an improved surveillance program, with the mosquito oviposition trap (MOT) method, integrating the actual monitoring locations to investigate the temporal and spatial distribution of Ae. albopictus abundance in an urban area of Shanghai, China from 2018 to 2019. A total of 133 monitoring units were selected for surveillance of Ae. albopictus density in the study area, which was composed of 14 subdistricts. The vector abundance and spatial structure of Ae. albopictus were predicted using a binomial areal kriging model based on eight MOTs in each unit. Results were compared to the light trap (LT) method of the traditional monitoring scheme.

Results: A total of 8,192 MOTs were placed in the study area in 2018, and 7917 (96.6\%) were retrieved, with a positive rate of $6.45 \%$. In $2019,22,715$ (97.0\%) of 23,408 MOTs were recovered, with a positive rate of $5.44 \%$. Using the LT method, 273 (93.5\%) and 312 (94.5\%) adult female Ae. albopictus were gathered in 2018 and 2019, respectively. The Ae. albopictus populations increased slowly from May, reached a peak in July, and declined gradually from September. The MOT positivity index (MPI) showed significant positive spatial autocorrelation across the study area, whereas LT collections indicated a nonsignificant spatial autocorrelation. The MPI was suitable for spatial interpolation using the binomial areal kriging model and showed different hot spots in different years.
\end{abstract}

Conclusions: The improved surveillance system integrated with a geographical information system (GIS) can improve our understanding of the spatial and temporal distribution of Ae. albopictus in urban areas and provide a practical method for decision-makers to implement vector control and mosquito management.

Keywords: Aedes albopictus, Mosquito oviposition trap, Binomial areal kriging model, Spatial distribution

\section{Background}

Aedes albopictus, also known as the Asian tiger mosquito, has invaded all continents except Antarctica during the last 30-40 years [1, 2]. Aedes albopictus is a primary human-biting pest species that significantly reduces

\footnotetext{
*Correspondence: zhuyiyi@scdc.sh.cn; wuhuanyu@scdc.sh.cn

${ }^{\dagger}$ Yibin Zhou and Hongxia Liu contributed equally to this work

1 Department of Infectious Disease Control, Shanghai Municipal Center for Disease Control and Prevention, Shanghai 200336, China

Full list of author information is available at the end of the article
}

the quality of life of infected persons, and is an invasive vector of major arboviral diseases, such as dengue, chikungunya, yellow fever, and Zika. In China, Ae. albopictus has adapted to low temperatures and is currently a primary nuisance pest and disease vector in the tropical and temperate regions of the country [3]. This species is present in regions where Aedes aegypti (Linnaeus) is absent [4], including Shanghai, and dengue is one of the most widely transmitted diseases carried by Ae. albopictus in China [5]. Aedes albopictus was reported to be the primary vector of several epidemics in Guangzhou original author(s) and the source, provide a link to the Creative Commons licence, and indicate if changes were made. The images or other third party material in this article are included in the article's Creative Commons licence, unless indicated otherwise in a credit line to the material. If material is not included in the article's Creative Commons licence and your intended use is not permitted by statutory regulation or exceeds the permitted use, you will need to obtain permission directly from the copyright holder. To view a copy of this licence, visit http://creativecommons.org/licenses/by/4.0/. The Creative Commons Public Domain Dedication waiver (http://creativeco mmons.org/publicdomain/zero/1.0/) applies to the data made available in this article, unless otherwise stated in a credit line to the data. 
Province (37,354 laboratory-confirmed cases) in 2014 [6] and Zhejiang Province (adjacent to Shanghai) in 2004 [7], 2009 [8], and 2017 [9].

The dengue case reported in 2017 was the first autochthonous dengue case in Shanghai in the last five decades [10]. Since then, three cases have been reported [11], and Ae. albopictus has been at the top of the list for vector control and surveillance in Shanghai. The public health service developed a monitoring system for Ae. albopictus to obtain information regarding its temporal evolution using the light trap (LT) and mosquito oviposition trap (MOT) methods in 2010. This system is used for surveillance of the Ae. albopictus population and biting rates based on data collected from subdistricts. However, a disadvantage of the surveillance network is that it does not integrate geographical information, and the subdistrict scale, which is usually $>3 \mathrm{~km}$, is large. As a result, only the regional average density of Ae. albopictus can be obtained with this monitoring system.

Geostatistical methods, which integrate the actual locations of samples, have been used to investigate the spatial distribution of mosquitoes [12] and several mosquitotransmitted diseases, including malaria $[13,14]$ and dengue fever $[15,16]$. Global and local indicators of spatial autocorrelation, such as Moran's $I$ [17] or local indicators of spatial association (LISA) [18], have been applied to study pests, including mosquitoes $[19,20]$. These indicators can detect hot spots of mosquito abundance and predict the significance of clustering and the effect of disease control [21]. Among the geostatistical methods, kriging interpolation [22] can predict the vector abundance in unsampled areas. Albieri et al. used kriging interpolation to predict the mosquito population distribution at the provincial and municipal scales in northern Italy [23], and Azil et al. used kriging to analyze the costs of dengue vector surveillance and control programs in Australia [24]. In addition, Giordano et al. created a more efficient larvicide control program for West Nile virus awareness campaigns in Canada by using kriging interpolation [25].

In China, the density of Ae. albopictus is usually assessed based on the Aedes-positive rate in MOT monitoring. MOT is a standard method of surveillance for the temporal and spatial distributions of container-inhabiting mosquitoes, including Ae. albopictus [26]. Current Aedes adult sampling methods, such as LTs, are laborintensive, expensive, and challenging to implement in large numbers [27]. As an alternative method, MOTs are artificial traps for Aedes egg collection. Compared to traditional traps, MOTs are more convenient and inexpensive to implement because they are easy to carry and do not require electricity or bait supplies. This method can detect the presence of gravid Aedes females with higher sensitivity, especially at low densities [28]. However, there have been rare reports [29] on the spatial interpolation of positive rates of Ae. albopictus.

The present study set out to evaluate the temporal and spatial distributions of Ae. albopictus using the MOT method, investigate the autocorrelation of Ae. albopictus abundance, and estimate Ae. albopictus abundance at non-sampling locations using the binomial areal kriging model [30] in an urban area of Shanghai, and identify hot spots and risk areas of high infestation. We created an improved surveillance program, which included the location of the traps and a change in the scale from 14 subdistricts to 133 monitoring units, for Ae. albopictus in an urban area of Shanghai from 2018 to 2019. Eight MOTs were applied in each unit to predict Ae. albopictus abundance and spatial structure at non-sampling locations. The LT method from the original monitoring scheme was also used and compared to the improved MOT method.

\section{Methods \\ Selection of the study area}

Shanghai is situated at $31^{\circ} 12^{\prime} \mathrm{N}$ latitude and $121^{\circ} 30^{\prime} \mathrm{E}$ longitude in the eastern part of the alluvial plain of the Yangtze Delta, adjacent to the Yangtze River estuarine and the East China Sea. It has four distinct seasons (spring from March to May, summer from June to August, autumn from September to November, and winter from December to February) and abundant precipitation, with a subtropical monsoon climate. The mean annual temperature in Shanghai is $\sim 17{ }^{\circ} \mathrm{C}$, and the mean annual precipitation is greater than $1100 \mathrm{~mm}$, with $53 \%$ occurring between June and September. The study area is situated in the center of Shanghai, China, with a total area of $37.37 \mathrm{~km}^{2}$, measuring $6.15 \mathrm{~km}$ from east to west and $11.93 \mathrm{~km}$ from south to north (Fig. 1). In 2018, the study region included 14 subdistricts with a resident population of $1,057,700$ [31].

\section{Meteorological data}

The monthly total precipitation and monthly mean maximum and minimum temperatures were calculated based on data from the China Meteorological Administration [32]. Weather variables were recorded at Xujiahui, which is located $\sim 2 \mathrm{~km}$ from the study area.

\section{Entomological survey}

The abundance of Ae. albopictus was analyzed using the MOT (Tian ${ }^{\circledR}$, Kaiqi Co., Ltd., Shanghai, China) and LT methods (Tian ${ }^{\circledR}$, Kaiqi Co., Ltd., Shanghai, China). The MOTs provide artificial breeding sites for containerbreeding mosquitoes, including Ae. albopictus, and positive MOTs were defined as those containing adults or eggs of Ae. albopictus. The LTs are baited with carbon 


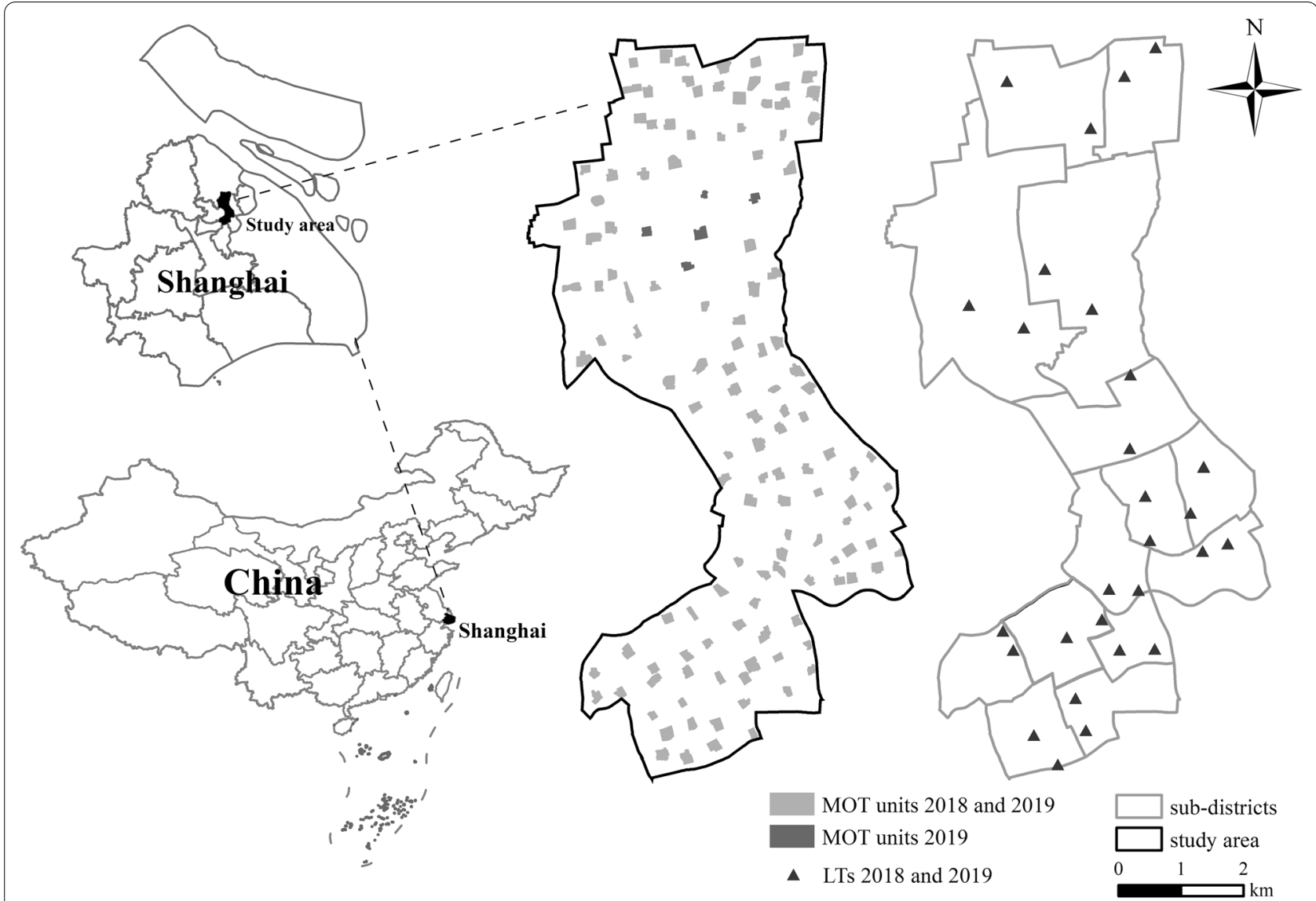

Fig. 1 Map of Shanghai, China, and locations of MOT monitoring units and LTs in the study area. MOT mosquito oviposition trap, LT light trap

dioxide to attract adult mosquitos, and the number of female adult mosquitoes captured was used as the index.

Each MOT [33] consists of a transparent cylindrical plastic jar (100 mm high, $70 \mathrm{~mm}$ diameter, $66 \mathrm{~mm}$ internal diameter) with a concave bottom (20 mm inward) and a black top cover with three conical openings $100 \mathrm{~mm}$ in diameter. When used as a collection container, white filter paper $70 \mathrm{~mm}$ in diameter, which is used as an egg deposition substrate, was placed inside the bottom of the MOT and $25 \mathrm{ml}$ of dechlorinated water poured into the jar to keep the paper moist but not submerged. MOTs were placed outdoors on grasslands, and kept away from direct sunlight, rain, and wind, at ground level by a skilled technician. To reduce competition among the MOTs, all of the traps were separated by a distance of more than $20 \mathrm{~m}$ and maintained unchanged until the end of the study.

In 2018, the MOTs were placed once a month between April and November. To enhance the data during the peak period, the frequency was increased in 2019 to once a week in week 20 , week 23 , week 25 , weeks $27-39$, and weeks 41-46. The MOTs that were removed, emptied, or interfered with for any reason were excluded from further analysis. After 4 days, each MOT was collected and species identification performed in the laboratory under a stereomicroscope. The MOT positivity index (MPI) was calculated as follows: $\mathrm{MPI}=$ number of Aedes-positive MOTs / total number of MOTs retrieved $\times 100 \%$.

The study area had 14 subdistricts containing 276 communities. Every two adjacent communities in one subdistrict were grouped into one polygon. When the number of communities in one subdistrict was odd, there was a polygon composed of three communities. We found a residential area with vegetative coverage in each polygon as the monitoring unit for the MOT (Fig. 1) and decided to place eight MOTs in each unit. The reasoning was as follows. First, the smallest subdistrict had six monitoring units. Second, this ensured that there were nearly 50 MOTs in each subdistrict, as noted in the Implementation Program of National Vector Surveillance launched by China's Center for Disease Control and Prevention. Third, the number of MOTs in each monitoring unit was consistent. In 2018, the center of the study area was under construction and MOTs could not be placed, 
so we put 128 units in the rest of the area (Fig. 1). The MPI values of the eight MOTs were used to represent the Ae. albopictus density of the unit. In 2019, we added five monitoring units in the middle of the study area, which led to a total of 133 monitoring units (Fig. 1).

Due to the low density of Ae. albopictus in April and the lower sensitivity of the LT compared with the MOT, the LT could not catch adult mosquitoes. Thus, on the third Wednesday of each month from May to November, the Center for Disease Control and Prevention set two LTs in every subdistrict throughout Shanghai as part of a citywide mosquito surveillance program. LTs were usually collected from 4 to $10 \mathrm{pm}$. The contents of these traps were sent to the laboratory for species identification, and only female Ae. albopictus were collected for data analyses.

The georeferenced positions of the MOT monitoring units and LTs in 2018 and 2019 are presented in Fig. 1.

\section{Cluster analysis}

The monitoring data were assigned to groups based on the type of trap and year of collection (i.e., MOT2018, MOT2019, LT2018, and LT2019) and analyzed. The total yearly collection of each LT and the mean MPI of each unit were calculated. Geostatistical analyses were conducted using the ArcGIS software (version 10.8; ESRI, Beijing, China) Spatial Statistics toolbox and data imported from Microsoft Excel 2019. Near table analyses were performed using the Generate Near Table tool to calculate the distance from each feature to its nearest neighboring feature based on the Euclidean distance.

We evaluated whether the mosquito abundances were spatially autocorrelated by calculating the incremental spatial autocorrelation (global Moran's $I$ at multiple distances). The global Moran's I [17] was tested using the permutation procedure based on feature locations and attribute values (yearly total collection from each LT and mean MPI of each unit) against the null hypothesis (the absence of spatial autocorrelation). This analysis identified the spatial patterns in the study area but did not indicate where such clusters occurred, which was determined by the local Moran's $I$ [18]. The local Moran's $I$ analysis was performed to assess the presence of hot spots with significant clusters, cold spots, and spatial outliers. For polygon MOT monitoring units, feature centroids were used in distance computations.

\section{Geostatistical analysis}

The kriging interpolation method [22] was used to quantify the spatial structure of the data and predict species abundance at unsampled locations. The binomial areal kriging model [30] is a geostatistical interpolation technique that extends the kriging theory to count data over polygons. In 1991, McNeill [34] derived the formulas for binomial kriging models. This step was performed using the ArcGIS 10.8 Geostatistical Analyst extension, including exploratory statistical analysis and variogram modeling. Each polygon of the input data contained a count (number of positive MOTs) and a population value (number of MOTs retrieved). The output was a figure predicting the positive rate and its standard error at each location.

A leave-one-out cross-validation method was used to determine whether the kriging interpolation provided reliable estimates at unsampled locations. The criteria used for accurate prediction in the cross-validation were as follows: root mean square standardized (RMSS) $\sim 1$, mean standardized (MS) $\sim 0$, and root mean square (RMS) approximately the average standard error (ASE).

\section{Digital map}

Digital maps of China and Shanghai from the National Catalogue Service for Geographic Information (National Bureau of Surveying \& Mapping, P.R. China), available at a scale of 1:1,000,000, were used as background for mapping. The shapefile of MOT monitoring units was produced by hand digitization of an aerial picture (Google Earth, May 2018; May 2019) in ArcGIS 10.8. The locations of LTs were georeferenced using GPS CHCNAV $\mathrm{X} 360 \mathrm{H}$ (WGS 1984 coordinate system) and later projected onto the UTM projection at the $51 \mathrm{~N}$ zone.

\section{Results}

\section{Mosquito collection}

In 2018, a total of 8192 MOTs were placed in the study area, and $7917(96.6 \%)$ of them were retrieved, with a positive rate of $6.45 \%$. In $2019,22,715$ (97.0\%) of 23,408 MOTs were recovered, with a positive rate of $5.44 \%$. LTs collected both male and female adult Ae. albopictus, with females constituting the majority; 273 (93.5\%) and 312 (94.5\%) adult female Ae. albopictus were gathered in 2018 and 2019 , respectively.

\section{Monthly distribution of Ae. albopictus}

The monthly mean temperature reached a peak in July $\left(33.2{ }^{\circ} \mathrm{C}\right)$ in 2018 and in August $\left(32.9{ }^{\circ} \mathrm{C}\right)$ in 2019 . The monthly precipitation was highest in August in both 2018 (230.5 mm) and 2019 (369.5 mm; Fig. 2). The total precipitation from May to October was almost twice as high in 2019 (1345.8 mm) as in 2018 (709.7 mm). In addition, the mean monthly maximum temperature from July to September was higher in $2018\left(31.9^{\circ} \mathrm{C}\right.$, Fig. 2a) than in 2019 (30.9 ${ }^{\circ} \mathrm{C}$, Fig. 2b).

As shown in Fig. 3, lower levels of oviposition were detected by MOTs in April 2018 and May 2019, with both MPIs peaking in July. In 2018, the monthly MPI peaked at 

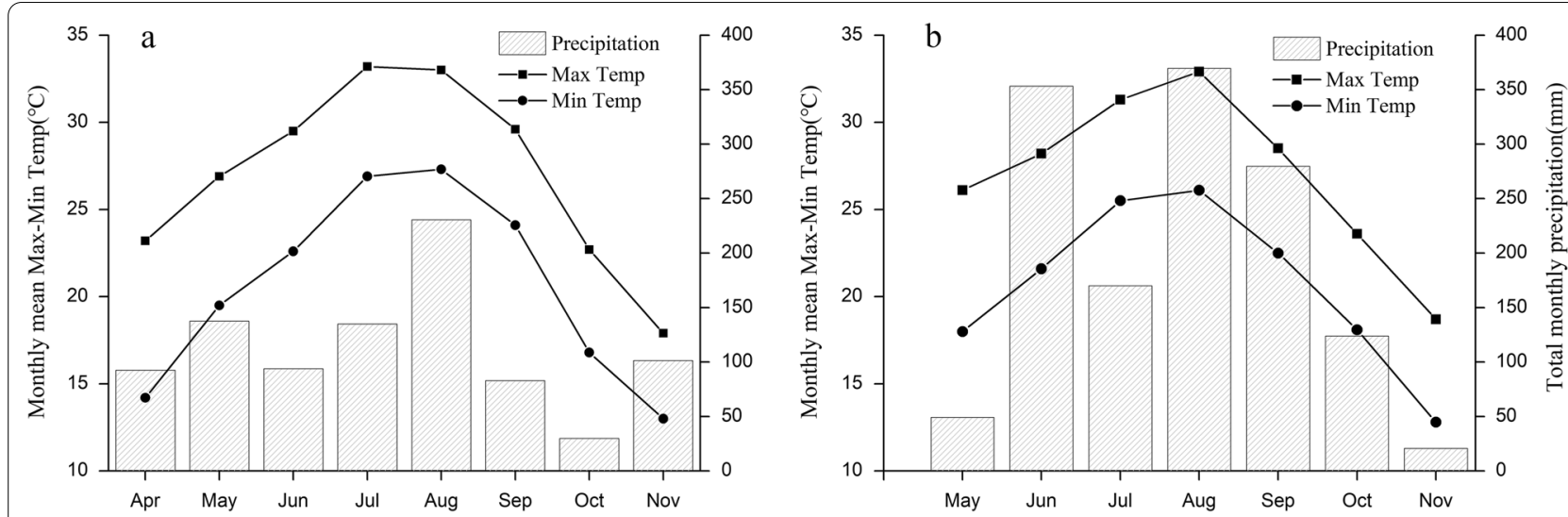

Fig. 2 Monthly maximum-minimum temperature and precipitation: a 2018, b 2019
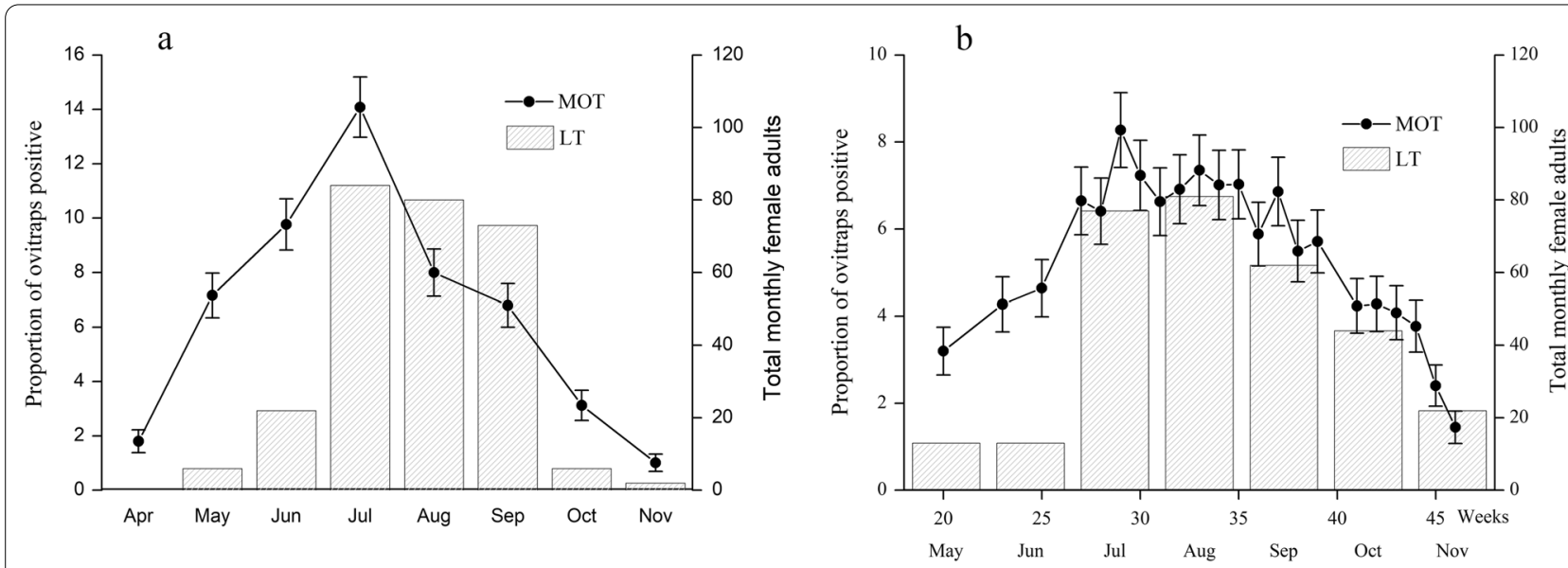

Fig. 3 The monthly MPI and number of female adult Ae. albopictus captured by LTs: a 2018, b 2019. MPI mosquito oviposition trap positivity index, LT light trap

$14.08 \%$, whereas in 2019 it peaked at $8.28 \%$ in the 29 th week (July). The MPI peak during the 2018 mosquito season was higher than the peak in the 2019 season. There was also a significant peak in the number of female adults collected by LTs from July to September in both years.

In 2018, the number of adult female Ae. albopictus collected by LTs peaked in July, which is consistent with the MPI. The number of female adults collected by LTs increased from May to July, reaching the highest values in July and August, and then decreasing in September. In general, the seasonal fluctuation curves in 2018 and 2019 indicated that Ae. albopictus populations in the urban area of Shanghai slowly increased from May, peaked in July, and declined gradually from September to October. The Spearman correlation coefficient $(r)$ between the monthly number of adult female Ae. albopictus collected by LTs and monthly MPI was $0.792(P=0.0334, d f=6)$ in 2018 and $0.756(P=0.0489, d f=6)$ in 2019.
Table 1 Results of the near table

\begin{tabular}{llll}
\hline Trap collection & MOT2018 & MOT2019 & LT2018 and 2019 \\
\hline $\begin{array}{l}\text { Numbers of units } \\
\text { Minimum distance }\end{array}$ & 128 & 133 & 28 \\
$\begin{array}{l}\text { (m) to the nearest } \\
\text { neighbor }\end{array}$ & 8.62 & 380.08 \\
$\begin{array}{l}\text { Maximum distance } \\
\text { (m) to the nearest } \\
\text { neighbor }\end{array}$ & & & \\
Mean distance $(m)$ & $179.37 \pm 98.87$ & $188.57 \pm 107.25$ & $702.05 \pm 263.50$ \\
\hline
\end{tabular}

MOT mosquito oviposition trap, $L T$ light trap

\section{Spatial distribution of MOTs and LTs}

The near table analyses produced the mean, minimum, and maximum distances between traps (Table 1). The mean distance between MOT monitoring units was 
$197.77 \mathrm{~m}$ in $2018,188.57 \mathrm{~m}$ in 2019 , and $702.05 \mathrm{~m}$ between LTs in both 2018 and 2019.

\section{Cluster analysis of Ae. albopictus abundance}

We performed incremental spatial autocorrelation analyses to evaluate the spatial autocorrelation of Ae. albopictus abundance across the study area. Only the MOT method demonstrated a significant positive spatial autocorrelation based on the mean MPI of each unit, which peaked at $651 \mathrm{~m}$ in 2018 and $528 \mathrm{~m}$ in 2019. The LT method did not show a significant spatial autocorrelation of Ae. albopictus abundance based on the total collected in each LT (Table 2).

Local Moran's $I$ was used to determine the locations of hot spots, cold spots, and spatial outliers. MOT2018, MOT2019, LT2018, and LT2019 had 13, 14, 2, and 1 clusters or outliers, respectively (Fig. 4).

Table 2 Results of incremental spatial autocorrelation analysis

\begin{tabular}{lllll}
\hline Trap collection & MOT2018 & MOT2019 & LT2018 & LT2019 \\
\hline Number of units & 128 & 133 & 28 & 28 \\
$\begin{array}{l}\text { The distance of peak } \\
\text { global Moran's I (m) }\end{array}$ & $651^{*}$ & $528^{*}$ & $680^{*}$ & $716^{*}$ \\
Global Moran's I & 0.297 & 0.279 & 0.227 & 0.303 \\
Z-score & 4.542 & 3.247 & 1.050 & 1.480 \\
$P$-value & $<0.001^{* *}$ & $<0.001^{* *}$ & $0.293^{* * *}$ & $0.139^{* * *}$
\end{tabular}

MOT mosquito oviposition trap, LT light trap

*Some units with no neighbors at this distance

**Significant positive spatial autocorrelation when $P<0.05$

***Random patterns when $P>0.05$

\section{Prediction of Ae. albopictus abundance at non-sampling} locations

We found that the mean MPI of each unit was suitable for kriging interpolation because it demonstrated significant positive spatial autocorrelation. There was no spatial autocorrelation for LT2018 and LT2019, and spatial interpolation was not permitted. We constructed the binomial areal kriging model using the yearly number of positive MOTs and the number of MOTs retrieved in each unit. Semivariograms created with the binomial areal kriging model showed spatial dependence (range) within $1900 \mathrm{~m}$ and $900 \mathrm{~m}$ for MOT2018 and MOT2019 (Figs. 5, 6), respectively, beyond which the semivariance

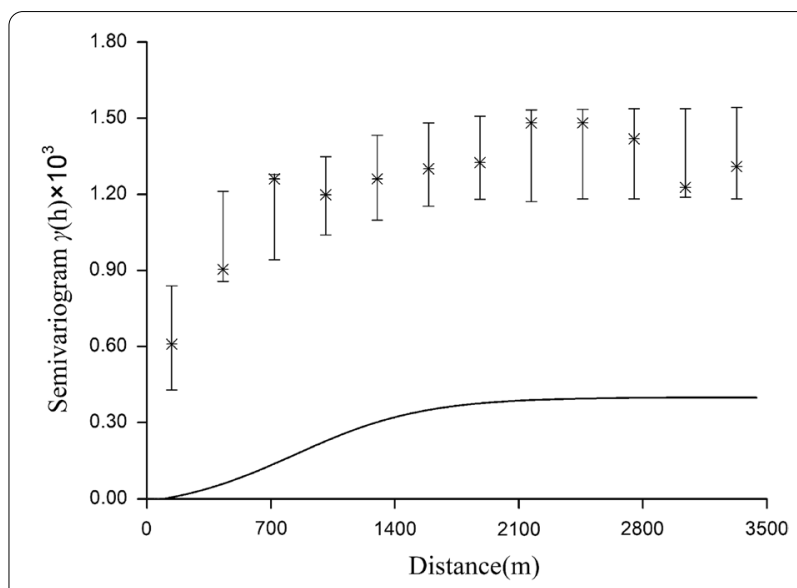

Fig. 5 Deconvoluted point semivariogram (line) and re-estimated empirical semivariogram values for polygons (stars) and their 90\% confidence intervals (vertical lines) for MOT2018

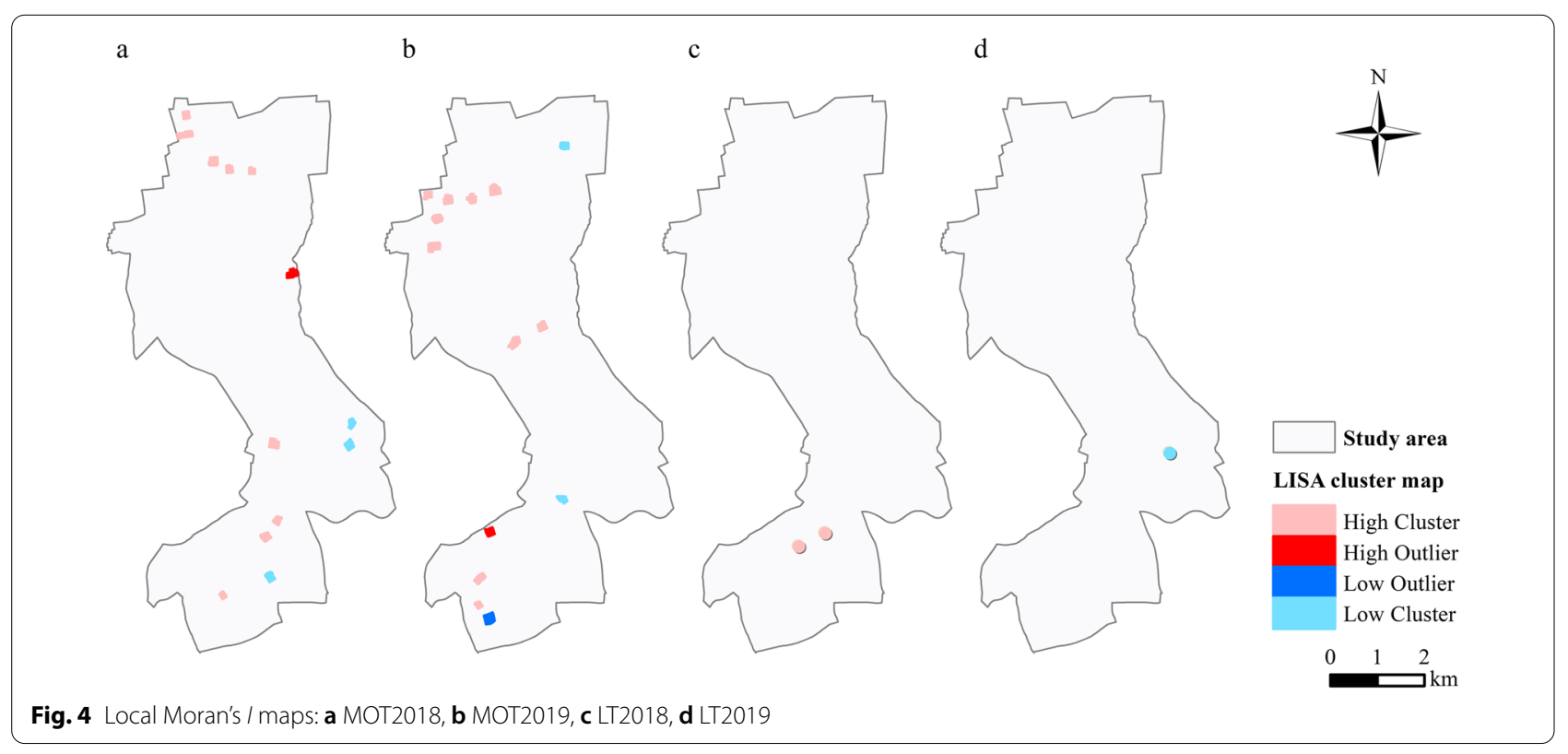




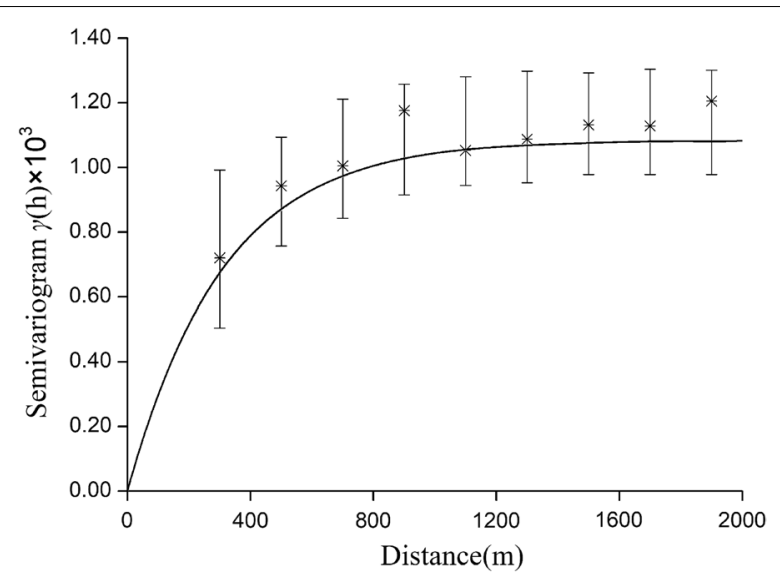

Fig. 6 Deconvoluted point semivariogram (line) and re-estimated empirical semivariogram values for polygons (stars) and their $90 \%$ confidence intervals (vertical lines) for MOT2018

remained constant. The best-fitting model for 2018 was the Gaussian model, and the exponential model best fit the data for 2019.

Prediction maps associated with the standard errors based on MOT2018 (Fig. 7) and MOT2019 (Fig. 8) data showed that the highest mosquito abundance and strong spatial clustering were in the southern and northern regions of the study area in 2018, and in the southern and central areas in 2019. The prediction of standard errors quantified the degree of data uncertainty for each location. According to this analysis, the prediction error was the lowest around where MOTs were set in the study area. Overall, the leave-one-out cross-validation statistics (Table 3) with the value of RMSS approaching 1 showed that the predicted models were reliable for map production.

\section{Discussion}

To the best of our knowledge, this study is the first to apply traps together with geostatistical methods to develop a routine mosquito surveillance program in China. Through this new program, a better understanding and more targeted Ae. albopictus control area was able to be achieved. We carried out this work in an urban area of Shanghai, where the human population density is high, with a higher blood-feeding rate of Ae. albopictus [35]. In addition, Ae albopictus is the dominant mosquito species, and sometimes the sole vector, in urban areas of China [36].

According to the Implementation Program of National Vector Surveillance launched by China's Center for Disease Control and Prevention, MOTs and LTs should be routinely applied for Ae. albopictus monitoring. Among the immature mosquito survey methods, MOTs have several advantages, including low cost, easy deployment, and noninvasive setup [37]. LTs have the advantage of simple operation, relatively objective monitoring results, and simultaneous monitoring at multiple sites [38]. In this program, each subdistrict, as a monitoring unit in

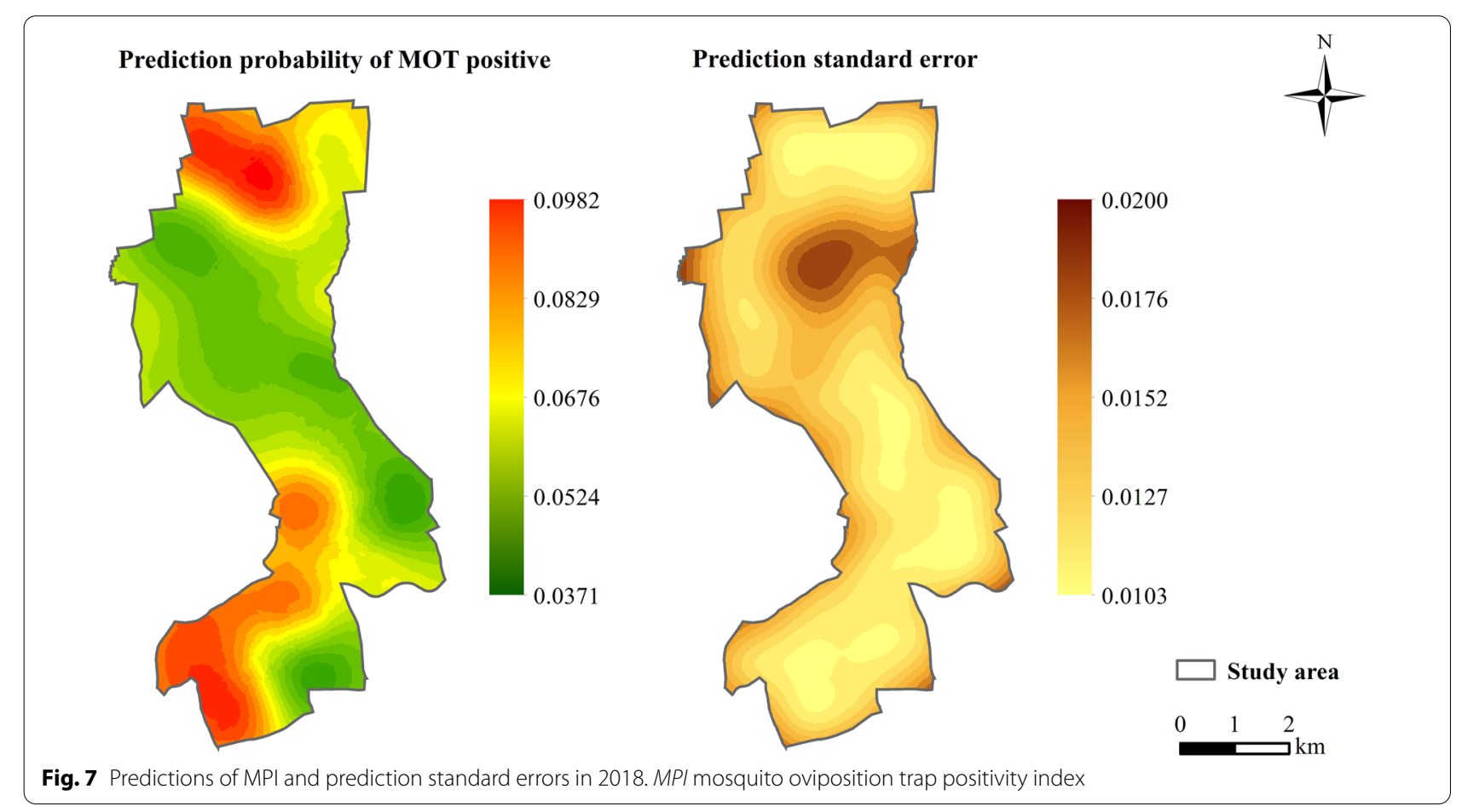




\section{Prediction probability of MOT positive}

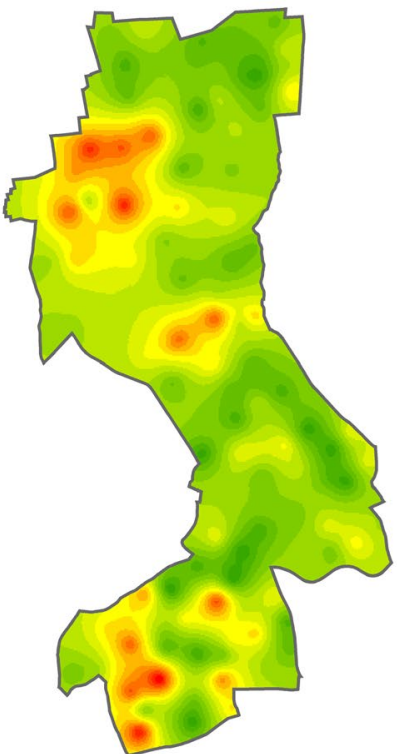

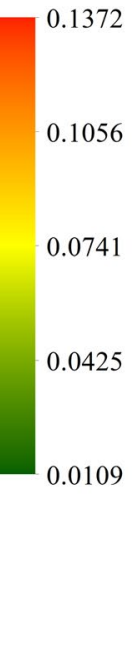
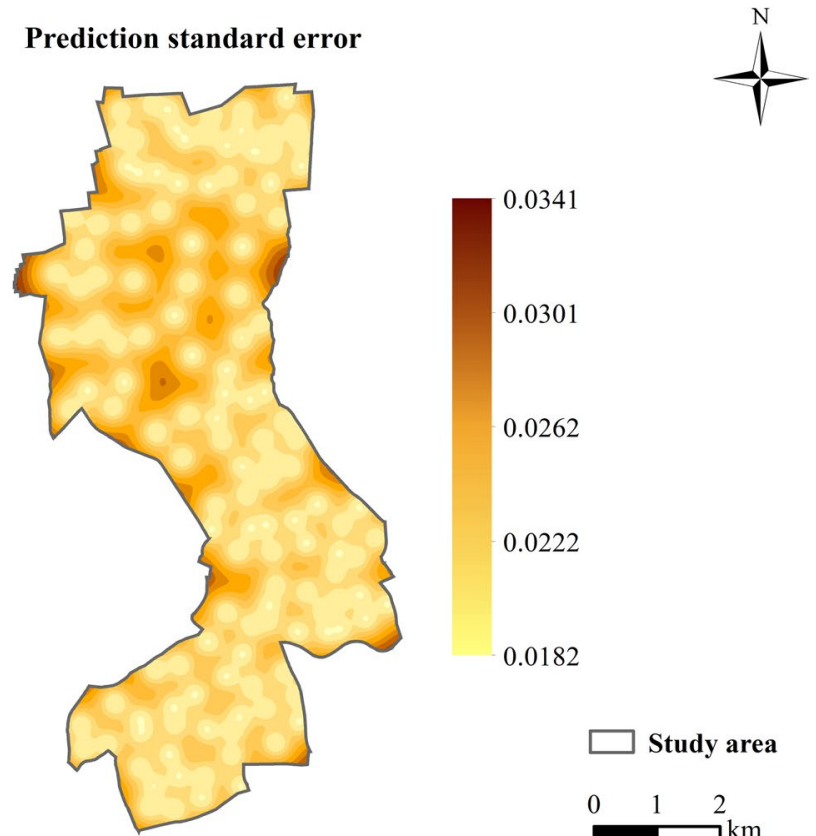

Fig. 8 Predictions of MPI and prediction standard errors in 2019. MPI mosquito oviposition trap positivity index

Table 3 Leave-one-out cross-validation statistics

\begin{tabular}{|c|c|c|c|c|c|c|}
\hline Year & Kriging type & Range (m) & RMS & MS & RMSS & ASE \\
\hline 2018 & Gaussian & 1910 & 0.0336 & 0.0023 & 0.9935 & 0.0338 \\
\hline 2019 & Exponential & 900 & 0.0326 & -0.0268 & 1.0085 & 0.0320 \\
\hline
\end{tabular}

RMS root mean square, $M S$ mean standardized, RMSS root mean square standardized, ASE average standard error

Shanghai, has a sample size of 2 LTs and 50 MOTs in a park and a residential area, but the spatial scale is limited for evaluating Ae. albopictus distribution [39, 40]. To obtain a more accurate seasonal and spatial distribution of the species in order to identify areas in need of effective target control, we developed an improved scheme dividing the subdistricts into 133 units. We also evaluated the accuracy of MOTs in combination with geostatistical analysis as a practical tool for monitoring the spatial distribution of Ae. albopictus in an urban area of Shanghai. Compared to the original system, the improved monitoring program obtained information on a unit scale rather than a subdistrict scale, including the spatial distribution of Ae. albopictus, and provided a finer spatial resolution to determine the need and allocation of effective resources for control.

The MPI peaked in July in both 2018 and 2019, whereas the LT collection peaked in July in 2018 and in August in 2019. However, the indices of LT remained high from July to September in both years. Consistent with the study by Gao et al., we found a significant correlation between monthly sampling yields [41]. The Ae. albopictus populations in the urban areas of Shanghai slowly increased from May, peaked from July to September, and declined after September, which coincides with the seasonal high temperatures and precipitation, and is also consistent with previous reports $[42,43]$.

In this study, we did not observe a spatial autocorrelation for the LT collections during different periods, possibly due to the small number or low density of LTs during these periods. As the spatial analyses used distances to establish neighbors, low numbers of neighbors may have resulted in lower statistical significance. The spread of $A e$. albopictus is limited by short-range flight, with a maximum distance of $600-800 \mathrm{~m}[44,45]$, which is close to the peak global Moran's I of MOT. Duncombe et al. [46] suggested the mosquito traps be placed $<1200 \mathrm{~m}$ from each other, but in this study the maximum distance between LTs and the nearest neighbor was $>1500 \mathrm{~m}$ (Table 1). The spacing of LTs was too large to detect spatial autocorrelation in the sample area [47]. In our study, two LTs were arranged in each subdistrict for different 
areas, which contributed to the different spatial density of the traps. More LTs evenly distributed with detailed geographical information will improve current surveillance in Shanghai.

According to our results, there was a significantly positive spatial autocorrelation of MOT2018 and MOT2019 across the study area, with a maximum distance of $600 \mathrm{~m}$ for the peak global Moran's I (Table 2). The semivariograms showed that mosquito collections from MOTs more than $1900 \mathrm{~m}$ in 2018 and $900 \mathrm{~m}$ in 2019 did not show a spatial autocorrelation (Figs. 7, 8). We focused on the spatial relationship with the risk of dengue fever, and we used the binomial areal kriging model because it is more suitable for MOT data, with the advantage of applying count data over polygons. The areal kriging model can make predictions and determine standard errors for all points within and between the input polygons by taking the sizes of the polygons into account [28, 48]. In this study, the estimated point semivariograms in Figs. 5 and 6 were different from the estimated empirical semivariograms for the polygons. Thus, the areal kriging can produce more accurate predictions than point kriging with values assigned to the polygon centroids $[30,49]$. The kriging maps revealed different oviposition hot spots in 2018 versus 2019, which may be attributed to the varying mosquito abundance and seasonal distribution from year to year due to changes in temperature, precipitation, and humidity $[50,51]$. In addition, the MOT2018 data may provide a reference for strict vector control in highdensity areas in 2019, causing a lower MPI density at these sites compared to other regions in 2019. Combined with the binomial areal kriging model, the improved surveillance scheme obtained a more accurate spatial distribution of Ae. Albopictus, and identified areas in need of effective target control.

Currently, some countries use a combination of mosquito monitoring and GIS. In Brazil, Noleto constructed a map to indicate sites with the largest number of collected eggs [52]. In Singapore, the GIS monitors the network of 2000 ovitraps placed island-wide to better understand vector trends and identify hot spots and risk areas where there is a danger of high Aedes infestation [53]. Using the GIS, an alert system was created from a synthesis of geospatial data on ovitrap indices in Hong Kong at the district level [54]. Compared to these countries, our new monitoring project can obtain data on the distribution of mosquitoes on a smaller scale, providing a basis for accurate allocation of public health resources and targeted control of Ae. albopictus density.

This surveillance project can be improved by combining spatial sampling or adding climate and environmental variables affecting Ae. albopictus abundance, which is recommended to improve the accuracy of the spatial interpolation [55]. The spatial sampling method with the machine learning random forest algorithm for climatic variables in Aedes abundance prediction can optimize the distribution of monitoring traps [56]. Future studies should include the preferred Normalized Difference Vegetation Index (NDVI) and the human population in spatial modeling of abundance, which will increase the accuracy and comprehensiveness of the model [24, 25].

\section{Conclusions}

In conclusion, an improved surveillance system with MOTs based on units can predict areas of Ae. albopictus abundance at non-sampling locations. This approach can improve our understanding of the spatial and temporal distribution of Ae. albopictus in urban regions of Shanghai and is a practical method for decision-makers to target vector control and management of mosquitoes with a finer spatial resolution. Future studies should explore the application of this monitoring program on a larger scale, and more data should be collected to validate this improved method.

\section{Abbreviations \\ MOT: Mosquito oviposition trap; LT: Light trap; MPI: MOT positivity index.}

\section{Acknowledgements}

The authors thank Zhu Zhenggang (Esri China), who is a GIS expert, for providing us with the map and help with the GIS technology.

\section{Authors' contributions}

Conceived and designed the experiments: HYW and YYZ. Performed the experiments: YBZ, HXL, PEL, and JZ. Analyzed the data: YBZ, SJY, and YYZ. Wrote the paper: YBZ, HXL, and YYZ. All authors read and approved the final manuscript.

\section{Funding}

This work was supported by Shanghai Municipal Health Commission (201940350). The funders had no role in study design, data collection and analysis, decision to publish, or preparation of the manuscript.

Availability of data and materials

All relevant data are within the paper.

\section{Declarations}

Ethics approval and consent to participate

This study did not involve vertebrate animals or human subjects.

Consent for publication

Written informed consent for the publication of the images was obtained from all individuals involved in this study.

\section{Competing interests}

The authors declare that they have no competing interests.

\section{Author details}

'Department of Infectious Disease Control, Shanghai Municipal Center for Disease Control and Prevention, Shanghai 200336, China. ${ }^{2}$ Key Laboratory of Geographic Information Science (Ministry of Education), East China Normal University, Shanghai 200241, China. 
Received: 6 February 2021 Accepted: 14 September 2021

Published online: 26 September 2021

\section{References}

1. Medlock JM, Hansford KM, Schaffner F, Versteirt V, Hendrickx G, Zeller $\mathrm{H}$, et al. A review of the invasive mosquitoes in Europe: ecology, public health risks, and control options. Vector Borne Zoonotic Dis. 2012;12:435-47.

2. Kotsakiozi P, Richardson JB, Pichler V, Favia J, Martins AJ, Urbanelli S, et al. Population genomics of the Asian tiger mosquito, Aedes albopictus: insights into the recent worldwide invasion. Ecol Evol. 2017;7:10143-57.

3. Li CX, Wang ZM, Dong YD, Yan T, Zhang YM, Guo XX, et al. Evaluation of lambda-cyhalothrin barrier spray on vegetation for control of Aedes albopictus in China. J Am Mosq Control Assoc. 2010;26:346-8.

4. Wu F, Liu Q, Lu L, Wang J, Song X, Ren D. Distribution of Aedes albopictus (Diptera: Culicidae) in northwestern China. Vector Borne Zoonotic Dis. 2011;11:1181-6

5. Wang YG, Lix X, Li CL, Su TY, Jin JC, Guo YH, et al. A survey of insecticide resistance in Aedes albopictus (Diptera: Culicidae) during a 2014 dengue fever outbreak in Guangzhou, China. J Econ Entomol. 2017:110:239-44.

6. Yang L, Chen Y, Yan H, Zhang P, Xu X, Tang B, et al. A survey of the 2014 dengue fever epidemic in Guangzhou, China. Emerg Microbes Infect. 2015;4:1-6.

7. Xu GZ, Dong HJ, Shi NF, Liu SJ, Zhou AM, Cheng ZH, et al. An outbreak of dengue virus serotype 1 infection in Cixi, Ningbo, People's Republic of China, 2004, associated with a traveler from Thailand and high density of Aedes albopictus. Am J Trop Med Hyg. 2007;76:1182-8.

8. Ling F, Fan WZ, Lin JF, Yan JY, LV HK, Fu T, et al. Epidemiological survey on a dengue fever outbreak in Yiwu, Zhejiang Province. Dis Surveill. 2010;25:757-9.

9. Yan H, Ding ZY, Yan JY, Yao WW, Pan JH, Yang ZN, et al. Epidemiological characterization of the 2017 dengue outbreak in Zhejiang, China and molecular characterization of the viruses. Front Cell Infect Microbiol. 2018:8:216-28.

10. Ji WJ. Successfully disposed of the first case of dengue fever in Shanghai. In: The compilation committee of Shanghai yearbook. Shanghai: Shanghai yearbook 2018; 2018. p. 453

11. Xu JM, Yang NY, Sun CW, Wang HJ, Leng PE, Zhu JF, et al. Evaluation on control measures and effects to Aedes albopictus at the epidemic sites of three local occurred dengue fever inflection cases in Baoshan District of Shanghai in 2018. Chinese Health Insecticide. 2019;6:275-9.

12. Espinosa M, Weinberg $D$, Rotela CH, Polop F, Abril M, Scavuzzo CM. Temporal dynamics and spatial patterns of Aedes aegypti breeding sites, in the context of a dengue control program in Tartagal (Salta Province, Argentina). PLoS Negl Trop Dis. 2016;10:e0004621.

13. Malvisi L, Troisi CL, Selwyn BJ. Analysis of the spatial and temporal distribution of malaria in an area of Northern Guatemala with seasonal malaria transmission. Parasitol Res. 2018;117:2807-22.

14. Zhou GF, Munga S, Minakawa N, Githeko AK, Yan GY. Spatial relationship between adult malaria vector abundance and environmental factors in western Kenya highlands. Am J Trop Med Hyg. 2007:77:29-35.

15. Russell RC, Ritchie SA. Surveillance and behavioral investigations of Aedes aegypti and Aedes polynesiensis in Moorea, French Polynesia, using a sticky ovitrap. J Am Mosq Control Assoc. 2004;20:370-5.

16. Barrera R. Spatial stability of adult Aedes aegypti populations. Am J Trop Med Hyg. 2011;85:1087-92.

17. Moran PA. Notes on continuous stochastic phenomena. Biometrika. 1950;37:17-23.

18. Anselin L. Local indicators of spatial association-LISA. Geogr Anal. 1995;27:93-115.

19. Castro MC, Kanamori S, Kannady K, Mkude S, Killeen GF, Fillinger U. The importance of drains for the larval development of lymphatic filariasis and malaria vectors in Dar es Salaam, United Republic of Tanzania. PLoS Negl Trop Dis. 2010;4:e693.

20. Zellweger RM, Cano J, Mangeas M, Taglioni F, Mercier A, Despinoy M, et al. Socioeconomic and environmental determinants of dengue transmission in an urban setting: an ecological study in Nouméa, New Caledonia. PLoS Negl Trop Dis. 2017;11:e0005471.
21. Manica M, Filipponi F, D'Alessandro A, Screti A, Neteler M, Rosà R, et al. Spatial and temporal hot Spots of Aedes albopictus abundance inside and outside a south European metropolitan area. PLoS Negl Trop Dis. 2016;10:e0004758.

22. Matheron G. Principles of geostatistics. Econ Geol. 1963;58:1246-66.

23. Albieri A, Carrieri M, Angelini P, Baldacchini F, Venturelli C, Zeo SM, et al. Quantitative monitoring of Aedes albopictus in Emilia-Romagna, Northern Italy: cluster investigation and geostatistical analysis. Bull Insectology. 2010;63:209-16.

24. Azil AH, Bruce D, Williams CR. Determining the spatial autocorrelation of dengue vector populations: influences of mosquito sampling method, covariables, and vector control. J Vector Ecol. 2014;39:153-63.

25. Giordano BV, Turner KW, Hunter FF. Geospatial analysis and seasonal distribution of West Nile Virus vectors (Diptera: Culicidae) in Southern Ontario, Canada. Int J Environ Res Public Health. 2018;15:614-31.

26. Service MW. Mosquito ecology: field sampling methods. 2nd ed. New York: Chapman \& Hall; 1993. p. 45.

27. Manica M, Rosà R, Della Torre A, Caputo B. From eggs to bites: do ovitrap data provide reliable estimates of Aedes albopictus biting females. Peer J. 2017:5:e2998.

28. Li Y, Su X, Zhou G. Comparative evaluation of the efficiency of the BG-Sentinel trap, CDC light trap and Mosquito-oviposition trap for the surveillance of vector mosquitoes. Parasit Vectors. 2016;9(1):446-54.

29. Garcez ACA, Mello MN, Ramos ELM, Protazio JMB, Farias PRS. Comparison of indicator Kriging and conditional simulation applied to spatial distribution of the vector Aedes aegypti. Bio Bra J. 2017;35:402-14.

30. Krivoruchko K, Gribov A, Krause E. Multivariate areal interpolation for continuous and count data. Proc Environ Ences. 2011;3:14-9.

31. Overview of Jing'an district. In: Shanghai: Jing'an District People's Government, Shanghai Municipality. http://www.jingan.gov.cn/. Accessed 17 Mar 2020.

32. Daily data set of climate data of China surface international exchange station (V3.0). In: China Meteorological Data Service Center. 2020. http:// data.cma.cn/dataService/cdcindex/datacode/SURF_CLI_CHN_MUL_ DAY_CES_V3.0/show_value/normal.html. Accessed 9 Mar 2020.

33. Lin LF, Lv WC, Cai SW. Guangdong disease prevent, China. Mosquito egg inducing trap. China patent CN2648815Y. 2004.

34. McNeill L. Interpolation and smoothing of binomial data for the southern African bird atlas project. South African Statist. 1991;25:129-36.

35. Valerio L, Marini F, Bongiorno G, Facchinelli L, Pombi M, et al. Host feeding patterns of Aedes albopictus (Diptera: Culicidae) in urban and rural contexts within Rome province, Italy. Vector Borne Zoonotic Dis. 2010;10:291-4

36. Yiji L, Fatmata K, Guofa Z, Santhosh P, Chunyuan L. Urbanization increases Aedes albopictus larval habitats and accelerates mosquito development and survivorship. PLoS Negl Trop Dis. 2014;8:e3301.

37. Service MW. Mosquito ecology: field sampling methods. 2nd ed. New York: Chapman \& Hall; 1993. p. 60.

38. Guo YH, Liu JL, Lu L, Chang SD, Zhang Y, Zhang J, et al. Comparative study on mosquito trapping effects of lamp trapping method and labor hour method. Chin J Vector Biol Control. 2012;23:529-32.

39. Sun J, Lu L, Wu H, Yang J, Xu L, Sang S, et al. Epidemiological trends of dengue in mainland China, 2005-2015. Int J Infect Dis. 2017:57:86-91.

40. Wu HX, Liu QY, Liu XB, Lu L, Guo YH, Yue YJ. Surveillance for Aedes albopictus in China, 2006-2013. Dis Surveill. 2015;30:310-5.

41. Gao Q, Cao H, Fan J, Zhang ZD, Jin S, Su F, et al. Field evaluation of Mosqovitrap, ovitrap and a $\mathrm{CO}_{2}$-light trap for Aedes albopictus sampling in Shanghai, China. Peer J. 2019;7:e8031.

42. Gao Q, Xiong CL, Zhou YB, Cao H, Jiang QW. Infestation status Aedes albopictus and related mosquito-borne infectious disease risk in central urban area in Shanghai. Chin J Epidem. 2016;37:600-5.

43. Yb Z, Zhao TY, Leng PE. Evaluation on the control efficacy of source reduction to Aedes albopictus in Shanghai, China. Chin J Vector Biol Control. 2009:20:3-6.

44. Honório NA, Silva Wda C, Leite PJ, Gonçalves JM, Lounibos LP, Lourençode-Oliveira R. Dispersal of Aedes aegypti and Aedes albopictus (Diptera: Culicidae) in an urban endemic dengue area in the State of Rio de Janeiro, Brazil. Mem Inst Oswaldo Cruz. 2003;98:191-8.

45. Liew C, Curtis CF. Horizontal and vertical dispersal of dengue vector mosquitoes, Aedes aegypti and Aedes albopictus, in Singapore. Med Vet Entomol. 2004;18:351-60. 
46. Duncombe J, Clements A, Davis J, Hu W, Weinstein P, Ritchie S. Spatiotemporal patterns of Aedes aegypti populations in Cairns, Australia: assessing drivers of dengue transmission. Trop Med Int Health. 2013;18:839-49.

47. Diuk-Wasser MA, Brown HE, Andreadis TG, Fish D. Modeling the spatial distribution of mosquito vectors for West Nile virus in Connecticut, USA Vector Borne Zoonotic Dis. 2006;6:283-95

48. What is areal interpolation? In: ArcGIS for Desktop. https://desktop.arcgis. com/en/arcmap/10.3/guide-books/extensions/geostatistical-analyst/ what-is-areal-interpolation.htm. Accessed 1 Dec 2020.

49. Kerry R, Goovaerts P, Smit IP, Ingram BR. A comparison of multiple indicator kriging and area-to-point Poisson kriging for mapping patterns of herbivore species abundance in Kruger National Park, South Africa. Int J Geogr Inf Sci. 2013;27:47-67.

50. Reeves WC, Hardy JL, Reisen WK, Milby MM. Potential effect of global warming on mosquito-borne arboviruses. J Med Entomol. 1994;31:323-32.

51. Zheng $X \mathrm{~L}$, Zhong DB, He YL, Zhou GF. Seasonality modeling of the distribution of Aedes albopictus in China based on climatic and environmental suitability. Infect Dis Poverty. 2019;8:98-106.

52. Oliveira Noleto JV, Moura do Nascimento Moraes HL, De Moura Lima T, Mendes Rodrigues JG, Tavares Cardoso D, Chaves Lima K, et al. Use of ovitraps for the seasonal and spatial monitoring of Aedes spp. in an area endemic for arboviruses in Northeast Brazil. J Infect Dev Ctries. 2020;14:387-93.

53. Aileen GT, Song RJ. The use of GIS in ovitrap monitoring for dengue control in Singapore. Dengue Bull. 2000;24:110-6.

54. Wong NS, Chi YL, Man KL, Shui SL, Lin H, et al. An alert System for informing environmental risk of dengue infections. Lect Notes Geoinformat Cartogr. 2007;6752:171-83.

55. Bailey AC, Gatrell TC. Interactive spatial analysis. UK: Longman Scientific \& Technical; 1995

56. Zheng JX, Xia S, Lv S, Zhang Y, Bergquist R, Zhou XN. Infestation risk of the intermediate snail host of Schistosoma japonicum in the Yangtze River Basin: improved results by spatial reassessment and a random forest approach. Infect Dis Poverty. 2021;10:74.

\section{Publisher's Note}

Springer Nature remains neutral with regard to jurisdictional claims in published maps and institutional affiliations.
Ready to submit your research? Choose BMC and benefit from:

- fast, convenient online submission

- thorough peer review by experienced researchers in your field

- rapid publication on acceptance

- support for research data, including large and complex data types

- gold Open Access which fosters wider collaboration and increased citations

- maximum visibility for your research: over $100 \mathrm{M}$ website views per year

At BMC, research is always in progress.

Learn more biomedcentral.com/submissions 